depended on the age or number of chemotherapy cycles. The 19 of 26 patients who attempted for pregnancy were leading to delivery (73\%). No one required infertility treatments. Mean of chemotherapy cycles and age is related to successful pregnancy. Conclusion: we showed patients with MOGCT could become pregnant and give birth if they desire. Advanced tumor stage was not the convincing factor for avoiding fertility preservation. Fertility sparing surgery with adjuvant chemotherapy is a safe treatment and results in high fertility rate.

Poster (010)

Basic/Translational Science

https://doi.org/10.3802/jgo.2021.32.S1.010

\section{Identification of the therapeutic and prognostic significance of STAU2 in ovarian cancer}

\section{Xiaoshi Liu*}

Ren Ji Hospital, School of Medicine, Shanghai Jiao Tong University Shanghai, China (xiaoshiliu@sjtu.edu.cn)

Objective: Of all the gynecologic cancers, ovarian cancer (OC) accounts for the most deaths worldwide. Factors such as chemotherapy and extensive abdominal metastasis may be important reasons leading to the high mortality of OC patients. Staufen homolog 2 (STAU2) is a member of the family of double-stranded RNA-binding proteins involved in cell fate decision by regulating mRNA transport, mRNA stability, translation, and ribonucleoprotein assembly. Past research have shown that STAU2, an anti-apoptotic protein, can participate in DNA replication and/or maintain genome integrity. However, the role of STAU2 in OC remains unknown.

Methods: GEPIA2 (http://gepia2.cancer-pku.cn/\#index) is an analysis tool containing RNA sequence expression data of 9,736 tumors and 8,587 normal samples from TCGA and the GTEx projects, using a standard processing pipeline. In this study, we used the "Single Gene Analysis" module of GEPIA2 to analyze the differential mRNA expression of tumor and normal tissues, perform pathological stage analysis, and analyze the prognosis value of claudins. The $\mathrm{p}$-value cutoff was 0.05 . The Human Protein Atlas (https://www.proteinatlas.org/) provides analysis of the secreted and membrane proteins, as well as an analysis of the expression profiles for all proteins targeted by pharmaceutical drugs and proteins implicated in cancer. In this study, we confirmed the expression of STAU2 in OC via immunohistochemistry using The Human Protein Atlas. Metascape (https://metascape.org/gp/index.html) includes analysis of bio-pathway enrichment, protein interaction network structure, and abundant gene annotation functions based on more than 40 public database. In this study, we performed an enrichment analysis of STAU2 and its most related genes by the Express Analysis module. TIMER (https://cistrome.shinyapps. io/timer/) is a comprehensive resource for systematical analysis of immune infiltrates across diverse cancer types. In this study, "Gene" module was used to evaluate the correlation between STAU2 level and the infiltration of immune cells. And we evaluated the correlation among clinical outcome and abundance of immune infiltrates expression using "Survival" module.

Results: STAU2 expression among serval cancers were analyzed, and we found the transcription level of STAU2 in OC tissues was significantly higher than normal tissues. We further confirmed that STAU2 in OC tissues overexpressed compared with normal tissues via immunohistochemistry. OC patients with higher expression of STAU2 were significantly associated with poorer overall survival $(\mathrm{p}=0.0014)$ and disease-free survival $(\mathrm{p}=0.039)$. Besides, the pathological stage of OC patients was closely related to the expression of STAU2 $(\mathrm{p}=0.0295)$, concluding that STAU2 may promote the progress of OC. GO/KEGG analysis showed the differential expression of STAU2 and its related genes were mainly associated with regulation of mRNA stability, endocytosis and ubiquitin mediated proteolysis. Then we found that CD8+ T cells expression (coefficient $=0.175$, $\mathrm{p}=5.64 \mathrm{e}-3$ ), CD4 $+\mathrm{T}$ cells (coefficient $=0.205, \mathrm{p}=1.13 \mathrm{e}-3$ ) and macrophages (coefficient $=0.353, \mathrm{p}=9.66 \mathrm{e}-9$ ) infiltration increased, while $B$ cells (coefficient $=-0.201, p=1.36 e-3$ ) and dendritic cells (coefficient $=-0.13, \mathrm{p}=3.98 \mathrm{e}-2$ ) infiltration decreased with high STAU2 expression. The Cox proportional hazard model showed CD4+ T cells $(\mathrm{p}=0.001)$, macrophages $(\mathrm{p}=0.001)$, neutrophils $(\mathrm{p}=0.030)$ and STAU2 expression $(\mathrm{p}=0.038)$ were significantly associated with the clinical outcome of OC patients. Conclusion: In summary, STAU2 may play a great influence on immune cell infiltration and may represent a valuable prognostic biomarker and therapeutic target for ovarian cancer.

Poster (011)

Gynecologic Pathology, Genetics and Epidemiology

https://doi.org/10.3802/jgo.2021.32.S1.011

\section{Molecular characterization and its correlation with tumor mutation burden in Chinese ovarian cancer patients}

\author{
Jia Jia Xu, Shoutai Ding, Huiling Chen, Chaoran Xia, \\ Zhixiang Yan, Peng Luo \\ Topgen Biological Medicine Technology Co., Ltd., Shanghai, China \\ (xujiajia@topgen.com.cn)
}

Objective: The breakthrough have been brought in therapy of ovarian cancer by poly(ADP-ribose) polymerase PARP inhibitor 